\title{
Heterodyne Standing-Wave Interferometer with Improved Phase Stability
}

\author{
Ingo Ortlepp ${ }^{1}$ · Jens-Peter Zöllner ${ }^{2} \cdot$ Ivo W. Rangelow $^{3} \cdot$ Eberhard Manske $^{1}$
}

Received: 21 December 2020 / Revised: 29 January 2021 / Accepted: 3 February 2021 / Published online: 23 June 2021

(c) The Author(s) 2021

\begin{abstract}
This paper describes a standing-wave interferometer with two laser sources of different wavelengths, diametrically opposed and emitting towards each other. The resulting standing wave has an intensity profile which is moving with a constant velocity, and is directly detected inside the laser beam by two thin and transparent photo sensors. The first sensor is at a fixed position, serving as a phase reference for the second one which is moved along the optical axis, resulting in a frequency shift, proportional to the velocity. The phase difference between both sensors is evaluated for the purpose of interferometric length measurements.
\end{abstract}

Keywords Standing wave $\cdot$ Interferometer $\cdot$ Heterodyne $\cdot$ Ultrathin photo diode

\section{Introduction}

Laser interferometers have been beneficially used for many years for high-precision length measurements in industry, precision engineering, and semiconductor processes. The applications include for example positioning stages, adjustment tasks or calibration purposes. However, in contrast to the simple optical principle, the real setup of commercial interferometers, usually based on the known "Michelson principle", is rather complex and requires elaborate adjustment during assembly. Therefore, those measurement devices are quite expensive and are only used for tasks with the highest demands for resolution, repeatability, and uncertainty. Furthermore, the potential for miniaturization is limited for those interferometer types because of the crosswise optical beam path, inherent to the Michelson principle, defining the minimum size of the optical components.

Ingo Ortlepp

ingo.ortlepp@tu-ilmenau.de

1 Institute of Process Measurement and Sensor Technology, Technische Universität Ilmenau, Ehrenbergstraße 29, 98693 Ilmenau, Germany

2 Microelectronic and Nanoelectronic Systems Group, Technische Universität Ilmenau, Ilmenau, Germany

3 Nanoscale Systems Group, Technische Universität Ilmenau, Ilmenau, Germany
In contrast to the complex optical setup, consisting of several voluminous, precision-made optical parts, standingwave interferometers (SWI) offer a simple linear structure with only a small number of components [1]. The basic homodyne principle requires only two transparent photo detectors, which build the actual interferometer, additional to the laser source and a measuring mirror [2].

The heterodyne SWI described in this paper utilizes an additional laser source of a similar wavelength to the first one for reducing the requirements for the transparent photo sensors, which are quite tight in the homodyne case. With the proposed structure, a heterodyne SWI offers an excellent potential for miniaturizing the complete interferometer down to a pair of transparent photo sensors with dimensions only slightly larger than the beam diameter. Additionally, these sensors can be manufactured with standard semiconductor technologies, enabling a cheap mass production of interferometer components.

\section{Principle of the Standing-Wave Interferometer}

\subsection{The Heterodyne Standing Wave}

Typically, the interference of electromagnetic waves is considered with two waves of the same wavelength, propagating in the same direction, resulting in a static 
interference pattern [3]. In contrast, the SWI utilizes the interference of two waves propagating and interfering in opposite directions. In the homodyne case, the counterpropagating wave is induced by simply inserting a mirror in the optical path. The resulting interference pattern forms a stationary standing wave, coupled to the inserted measuring mirror [4] (see Fig. 1).

In the setup described in this paper, the counter-propagating wave is generated by a second laser source and hence in general, it is independent of the first wave considering wavelength and phase. As in the homodyne case, the two electromagnetic waves interfere in opposite directions and form an optical standing wave. In contrast to the homodyne standing wave, which has a static intensity profile coupled to the measuring mirror, the intensity profile of the heterodyne standing wave will move along the optical axis with a velocity $\dot{z}_{\mathrm{SW}}$ depending on the wavelength difference of the two laser sources [5] (see Fig. 2).

Assuming the same amplitude and a phase angle of zero, the electromagnetic waves of Fig. 2 can be described as

$$
\begin{aligned}
& E_{1}=\hat{E} \cos \left(2 \pi f_{1}\left(\frac{z}{c}-t\right)\right), \\
& E_{2}=\hat{E} \cos \left(2 \pi f_{2}\left(-\frac{z}{c}-t\right)\right)
\end{aligned}
$$

with the electric field intensity $E$, amplitude $\hat{E}$, frequency of the laser source $f$, optical axis $z$, speed of light $c$, and time $t$. When the two electromagnetic waves interfere in opposite directions, the final field intensity results from the superposition of $E_{1}$ and $E_{2}$ :

$E_{\mathrm{res}}=E_{1}+E_{2}$.

By applying the addition theorem for cos, Eq. (3) can be rewritten as

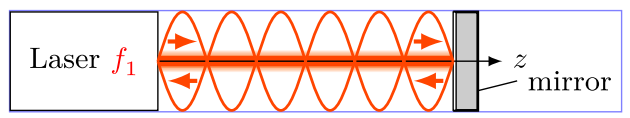

Fig. 1 Stationary homodyne standing wave between a laser source and a measuring mirror. The intensity profile of the standing wave is phase coupled to the mirror surface, so moving the mirror will also shift the standing wave along the optical axis $z$

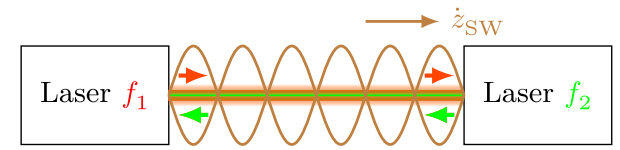

Fig. 2 Heterodyne standing wave between two laser sources of different frequencies $f_{1}, f_{2}$. The intensity profile of the standing wave is moving with a velocity $\dot{z}_{\text {SW }}$ along the optical axis $z$

$$
\begin{aligned}
E_{\mathrm{res}}= & 2 \hat{E} \cos \left(\frac{2 \pi\left[\left(f_{1}-f_{2}\right) \frac{z}{c}-\left(f_{1}+f_{2}\right) t\right]}{2}\right) \\
& \cdot \cos \left(\frac{2 \pi\left[\left(f_{1}+f_{2}\right) \frac{z}{c}-\left(f_{1}-f_{2}\right) t\right]}{2}\right)
\end{aligned}
$$

As the two laser sources have almost the same frequencies $f_{1} \approx f_{2}$, the terms containing $f_{1}-f_{2}$ can be considered as an amplitude modulation for the terms containing the much higher frequency $f_{1}+f_{2}$ [3]. Thus, the electric field intensity is not constant over space and time in a heterodyne standing wave. Instead, the heterodyne standing wave is moving along the optical axis $z$.

State-of-the-art photo detectors are too inert to detect electromagnetic waves at optical frequencies directly. Therefore, the mean energy flux density, the intensity of the wave, calculated by the magnitude of the Poynting vector, is detected. The intensity $I_{\mathrm{SW}}$ of a heterodyne standing wave at the position $z=0$ is therefore given by

$I_{\mathrm{SW}} \sim \hat{E}^{2}\left(1+\cos \left(2 \pi\left(f_{1}-f_{2}\right) t\right)\right)$.

Hence, at a fixed position, the intensity of the standing wave is modulated with the difference frequency $f_{1}-f_{2}$ of the two laser sources, called beat frequency $f_{\mathrm{b}}$. This modulation is caused by the motion of the standing wave along the optical axis with a velocity of

$\dot{z}_{\mathrm{SW}}=f_{\mathrm{b}} \frac{\lambda}{2}$.

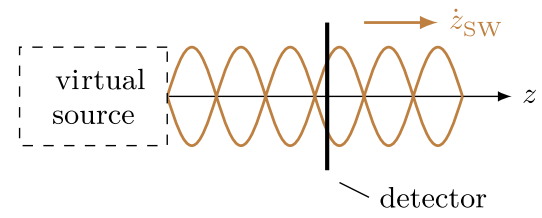

Fig. 3 Heterodyne standing wave, moving along the optical axis, virtually emitted by a single laser source (compare Fig. 2). The intensity profile can be detected by a thin and transparent photo detector 
So between two laser sources, placed diametrically opposed on the optical axis, emitting towards each other, an optical standing wave arises, moving with the given speed $\dot{z}_{\mathrm{SW}}$, causing a periodic modulation of the local beam intensity with the frequency $f_{\mathrm{b}}$. Depending on $f_{\mathrm{b}}$, the standing wave seems to be emitted by a virtual source and can be detected by a thin and transparent photo detector (see Fig. 3 and next section).

\subsection{Detection of the Optical Standing Wave}

The intensity profile of the standing wave can be detected by a photo sensor, which is inserted into the optical beam path. As this sensor must not affect the standing wave, it has to be transparent to further allow the interference of the two opposed laser beams. Because of this and further requirements, special photo detectors are necessary, called standing-wave sensors (SWSs).

When such a sensor is at rest, the modulated intensity of the standing wave will induce an sin-shaped sensor signal with the beat frequency $f_{\mathrm{b}}$. For a sensor moved with a velocity $\dot{z}_{\text {Sws }}$ along the optical axis, the current sensor position for a given time $t$ can be calculated by

$z=\dot{z}_{\mathrm{SWS}} t+z_{0}$.

Inserting Eq. (7) into Eq. (4) and assuming the starting point $z_{0}=0$ leads to

$$
\begin{aligned}
& I_{\mathrm{SW}} \sim \\
& \quad \hat{E}^{2}\left(1+\cos \left(2 \pi\left[\left(f_{1}-f_{2}\right)-\left(f_{1}+f_{2}\right) \frac{\dot{z}_{\mathrm{SWS}}}{c}\right] t\right)\right) .
\end{aligned}
$$

The amplitude modulated sensor signal is therefore affected by a Doppler shift with a proportion of

$f_{\mathrm{D}}=-\left(f_{1}+f_{2}\right) \frac{\dot{z}_{\mathrm{SWS}}}{c}$

resulting in an overall detected frequency of

$f_{\text {Sen }}=f_{\mathrm{b}}+f_{\mathrm{D}}$.

So, for a given beat frequency $f_{\mathrm{b}}$, it is possible to calculate the Doppler shift $f_{\mathrm{D}}$ from $f_{\text {Sen }}$. Based on the value and the sign of the Doppler shift, the moving direction and velocity $\dot{z}_{\text {SWS }}$ of the photo detector can be calculated for known $f_{1}$ and $f_{2}$.

In summary, the heterodyne standing-wave interferometer (HSWI) in its fundamental implementation consists only of two diametrically opposed laser sources and a single transparent standing-wave sensor. Based on the detected frequency, the moving direction and velocity of the sensor can be determined, allowing length measurements inside the space between the two laser sources. As the principle contains no further parts, the HSWI has a simple linear structure with a cross section only slightly larger than the laser beam diameter, see Fig. 4.

\section{State of the Art}

\subsection{Heterodyne Laser Source}

The principle of the HSWI requires two laser sources with well-defined frequencies $f_{1}$ and $f_{2}$. The obvious solution, utilizing two independent stabilized laser sources is not sufficient in practice. The reason is the long-term drift of the frequency of every single laser. Because of that drift, the beat frequency $f_{\mathrm{b}}$ is not constant over time. So, it can be possibly zero if accidentally $f_{1}=f_{2}$. Or it can exceed the cut-off frequency of the SWS if $f_{\mathrm{b}}>f_{\mathrm{c}}$. It can even reverse the moving direction of the standing wave, in the case $f_{\mathrm{b}}$ changes sign.

Therefore, a coupling of the two laser frequencies is necessary. This can be achieved by different approaches, the most common ones are splitting the emission line of the laser by an external magnetic field (Zeeman effect [6]), modulating the laser beam by an acousto-optic modulator (AOM) [7] or using a two-mode helium-neon $(\mathrm{He}-\mathrm{Ne})$ laser source [8].

In a two-frequency He-Ne Zeeman laser, the maximum frequency difference is up to $f_{\mathrm{b}}=3 \mathrm{MHz}$ [9]. As the frequency difference $f_{\mathrm{b}}$ defines the maximum velocity of the sensor in the HSWI (Eq. (6) and (9)), there is a restriction of approx. $\dot{z}_{\text {Sws }}<0.83 \mathrm{~m} \mathrm{~s}^{-1}$, which limits the fields of applications. Furthermore, the two frequencies are coaxial and have to be separated, which can only be achieved imperfectly. The resulting polarization leakage leads to non-linearities in the interferometer signal [10].

The widely used two-frequency laser system based on the frequency modulation of one or two He-Ne lasers with an AOM can be implemented with one or two AOMs. With one $\mathrm{AOM}$, the minimum beat frequency is approx. $f_{\mathrm{b}}>20 \mathrm{MHz}$, which raises enormous demands for the utilized SWSs. For creating an arbitrary beat frequency, two AOMs are necessary. However, the cost of such a system is enormous.

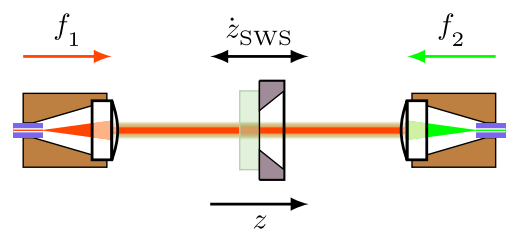

Fig. 4 Fundamental principle of a heterodyne SWI with two coaxial, diametrically opposed laser sources (here realized as fiber collimators) and a moving SWS in between 
Creating two frequencies for the HSWI by a two mode $\mathrm{He}-\mathrm{Ne}$ laser [11] results in an extremely high beat frequency up to $f_{\mathrm{b}} \approx 750 \mathrm{MHz}$. This raises enormous challenges in the field of high-frequency signal processing. Furthermore, the two frequencies are coaxial and have to be separated, too.

Most of the heterodyne interferometers are free beam interferometers, where the laser beams are directly guided into the optical setup. This causes additional effort to be taken for a stable beam guidance with additional optical parts. Furthermore, it is hard to separate the laser source from the sensitive interferometer to avoid heat transfer and other disturbances. Because of these problems, fiber coupled laser sources are usually used for convenience. They offer more flexibility, a smaller form factor in critical optical setups, and reduce heat dissipation to almost zero. However, the two heterodyne frequencies should not be guided in a common fiber, as this can lead to polarization leakage and frequency mixing due to mechanical stress inside the fiber $[12,13]$.

To enable a separated generation of the two laser beams as well as a separate fiber coupling, two-frequency coupled $\mathrm{Ne}-\mathrm{Ne}$ laser sources with a free adjustable beat frequency according to $[14,15]$ are used in this project. The advantages of using stabilized He-Ne lasers are a narrow line width of down to $1 \mathrm{~Hz}$ and a visible wavelength of $632.8 \mathrm{~nm}$, allowing the usage of standard optical and optoelectronic components. The setup consists of two $\mathrm{He}-\mathrm{Ne}$ lasers where one of them is the master laser, which is frequency-stabilized by the two-mode stabilization technique [16], defining the absolute frequency $f_{1}$ with a stability of $\pm 5 \times 10^{-9}$ [17]. This master laser is the frequency reference for the slave laser. The frequency coupling between master and slave is realized by a phase-locked loop (PLL), which controls the frequency difference, determined by the interference of the two emitted laser beams on a photo diode (Fig. 5). The reference signal for the PLL can be generated by a crystal resonator or a frequency generator, allowing a tunable, well-defined and long-term stable beat frequency $f_{\mathrm{b}}$.

The beams of the master and the slave laser are separately coupled into an optical fiber and guided to the HSWI, thus preventing sources of non-linearities like frequency mixing

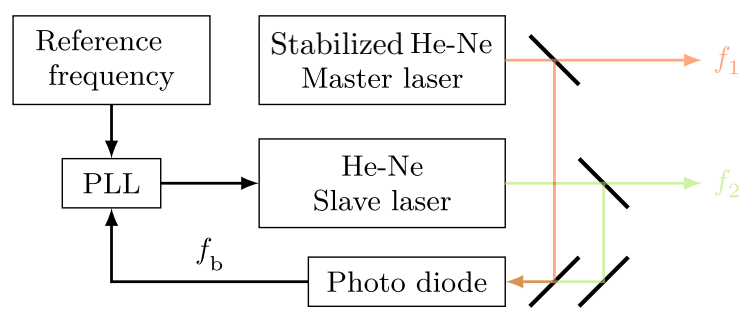

Fig. 5 Principle of the heterodyne laser source. The master laser is a two-mode stabilized $\mathrm{He}-\mathrm{Ne}$ laser and the slave is controlled by a PLL, guaranteeing a tunable and stable beat frequency $f_{\mathrm{b}}$. [5] and polarization leakage in the interferometer setup, reducing thermal effects, etc., as described above. Furthermore, the two laser beams can be adjusted separately inside the interferometer setup.

\subsection{Standing-Wave Sensor}

As described earlier, special photo detectors are required for detecting the local intensity of a standing wave.

The existence of optical standing waves was already shown by Wiener [18] in 1890 . There, a glass plate was covered with a thin, photo-sensitive layer to detect the periodic intensity of a homodyne standing wave in front of a mirror.

The first proposal for a standing-wave interferometer for length measurements was made by Büchner [1] in 1983. In his patent, the basic structure of a homodyne standing-wave interferometer with laser source, measuring mirror and two serially arranged, $90^{\circ}$ phase shifted sensors is described.

The particular requirements for an SWS in a standingwave interferometer are a sufficient transparency, a thickness smaller than the optical wavelength, an excellent flatness, and a low reflectivity [19].

Adequate electro-optical sensors for detecting a standing wave were described by Silvertooth [20] and later by Sasaki [21]. Sasaki's SWS is made of a quartz plate, which is coated with a thin, transparent silicon layer. In this sensor, the two photo diodes for forward-backward counting are arranged side-by-side. The phase shift between both sensors is achieved by local etching of the quartz substrate, changing the optical path length for one sensor by $\pi / 2$.

An SWS made of amorphous silicon on a glass plate is described by Bunte and Mandryka [2, 22]. Here, the two sensors for obtaining phase-shifted quadrature signals are arranged consecutively along the optical axis by successively depositing appropriate doped silicon layers on the carrier substrate, resulting in two stratified p-i-n photo diodes.

The described SWSs and their application in standing waves for length measurements use homodyne standing waves, which occur after the reflection of a laser beam at a mirror. The principle and application of a heterodyne standing-wave interferometer was first described in [5]. For a heterodyne setup according to Fig. 4, there is an additional demand for a fast electro-optical response of the photo sensor in order to ensure a reliable detection of the moving standing wave. The requirements, manufacturing, structure, and properties of such sensors are extensively described in $[5,19,23]$. Figure 6 shows the cross section of such a transparent SWS suitable for an HSWI and Fig. 7 shows an exemplar, used in this project.

The minimal implementation of the HSWI with a single SWS [5], as shown in Fig. 4, lacks a reference for the beat frequency $f_{\mathrm{b}}$. In this setup, $f_{\mathrm{b}}$ is detected inside the heterodyne laser source (Fig. 5). Due to possible 


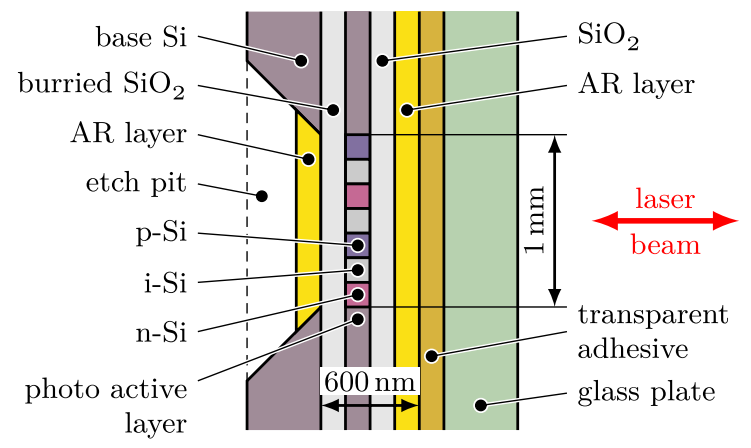

Fig. 6 Cross section of the SWS, based on a silicon-on-insulator wafer. For transparency, the base silicon is etched in the region of the photosensitive $\mathrm{p}-\mathrm{i}-\mathrm{n}$ diode. Additionally, the front and rear side of the sensor is anti-reflection (AR) coated. The glass plate stabilizes the ultra-thin membrane (thickness $\approx 600 \mathrm{~nm}$ ). [5]

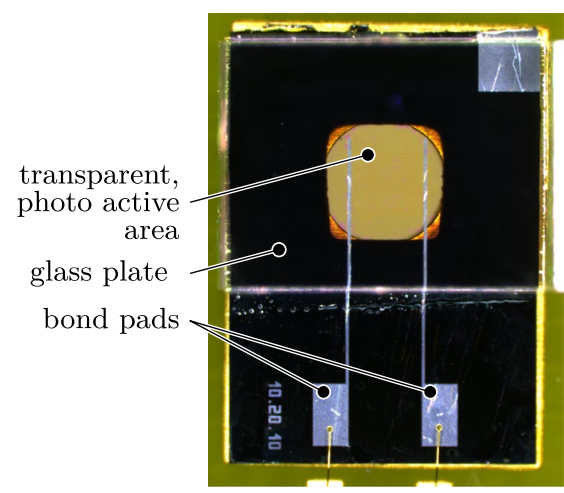

Fig. 7 Detailed view of the SWS with transparent, photo active area and stabilizing glass plate. View along the optical axis

disturbances in the transmission of the laser light to the actual interferometer, mainly because of fluctuations of the refractive index in the transmission path (optical elements, air, fiber), a varying phase shift between the beat frequency determination and the interferometer may occur. These fluctuations can be long-term drifts as well as high-frequency phase noise, in particular, when using fiber coupled laser sources. The phase of the standing wave in the HSWI is the reference for the length measurement. Therefore, any phase variations directly affect the calculated position of the SWS in the interferometer, because with a single SWS, it is impossible to distinguish between phase shifts due to transmission errors and phase shifts due to sensor movement and the resulting Doppler shift.

To overcome this problem, an advanced principle is used, described in the next section.

\section{Principle of Operation}

\subsection{Optical setup}

As described above, a single-SWS setup is not suitable for reliable length measurements because of the uncertainty for the phase of the reference frequency $f_{\mathrm{b}}$. The problem can be solved by adding a second SWS into the optical beam path. In contrast to the first SWS, the second sensor is placed at a fixed position and serves as a reference for determining the current phase of the beat frequency, see Fig. 8. The sensor is at rest at all times ( $\left.\dot{z}_{\text {SwS }}=0\right)$, hence it is not affected by the Doppler shift. From Eq. (9) and (10) it can be deduced that for the second sensor is $f_{\mathrm{Sen}}=f_{\mathrm{b}}$.

Any phase shift in the transmission between laser source and the actual interferometer will affect both sensors, the moving one as well as the second, fixed, sensor. In this implementation, it is now possible to calculate the phase difference between the moving and the fixed SWS, effectively measuring the distance between them, unaffected by phase displacements in the laser light transmission from the source to the interferometer.

\subsection{Signal Processing}

The principle described earlier requires determining the phase difference between the signals of the two SWSs: $s_{\mathrm{b}}$ of the fixed SWS as reference for $f_{\mathrm{b}}$ and $s_{\mathrm{m}}$ of the moving SWS.

The phase difference between two signals can be determined by different techniques:

- measuring the time difference between the zero crossings of both signals,

- calculating a Fourier transform of both signals,

- approximation by an ideal signal,

- calculating the cross correlation between both signals and

- transferring both signals to the base band and executing an arctan demodulation.

In this project, the transfer to the base band is used and realized by a lock-in technique as described in [25]. For that purpose, both sensor signals are mixed with an

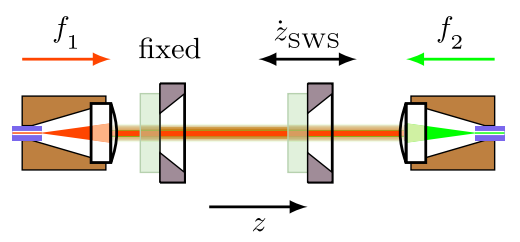

Fig. 8 Optical setup of a two-SWS HSWI with a fixed sensor as a reference for $f_{\mathrm{b}}$ and a moving measuring sensor. [24] 
artificial signal of a local oscillator with a frequency $f_{\mathrm{LO}}$ and the resulting product is low pass filtered afterwards.

For that purpose, the sensor signal

$s_{\mathrm{m}}=\hat{s}_{\sim} \cos \left(2 \pi f_{\mathrm{Sen}} t+\delta_{\mathrm{S}}\right)$

of the SWS with the phase $\delta_{\mathrm{S}}$ and the signal

$s_{\mathrm{LO}}=\hat{s}_{\mathrm{LO}} \cos \left(2 \pi f_{\mathrm{LO}} t+\delta_{\mathrm{LO}}\right)$

of the local oscillator (LO) with the phase $\delta_{\mathrm{LO}}$ are mixed. By lowpass filtering the resulting signal, higher-order mixing products are eliminated.

$s_{\mathrm{m}} s_{\mathrm{LO}}=\cos \left(2 \pi\left(f_{\mathrm{Sen}}-f_{\mathrm{LO}}\right) t+\delta_{\mathrm{S}}-\delta_{\mathrm{LO}}\right)$.

If the mixing is carried out with two LO of different phase angles, it is possible to generate two $90^{\circ}$ phase-shifted quadrature signals. These signals can be fed into an arctan demodulation, allowing to determine the phase difference $\delta_{\mathrm{D}}$. Figure 9 shows the algorithm of the described principle.

The computations in this section are done by a systemon-chip. On this board, the signals of the SWSs are A/D converted and then processed in the FPGA part of the system. The calculated fringe and phase information $\left(\delta_{\mathrm{D}}\right)$ as well as a timestamp are transferred to the operating system and made available via an Ethernet connection to a client computer (see Fig. 10). The system and the algorithm are further described in [24].

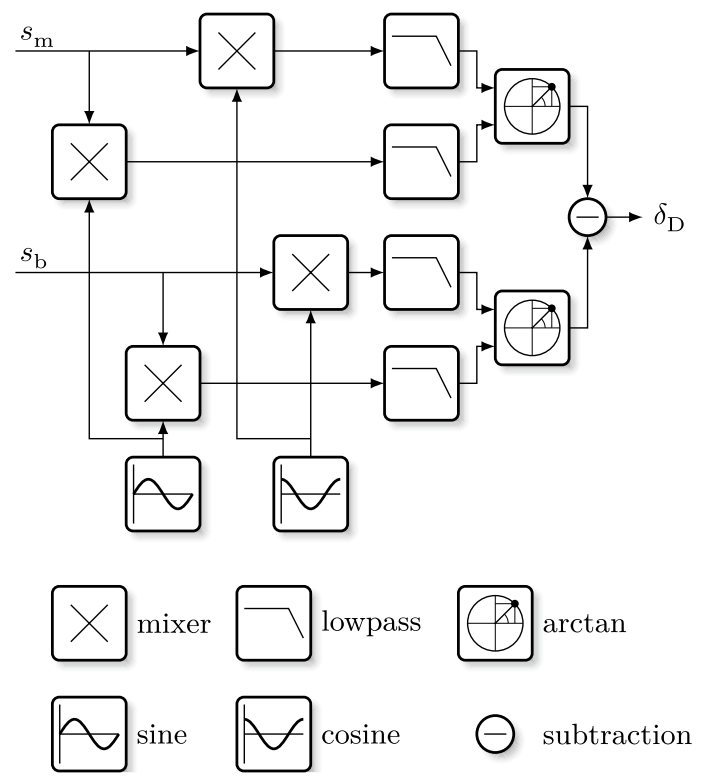

Fig. 9 Signal processing of the HSWI for determining the phase difference $\delta_{\mathrm{D}}$ between $s_{\mathrm{m}}$ and a reference signal $s_{\mathrm{b}}$

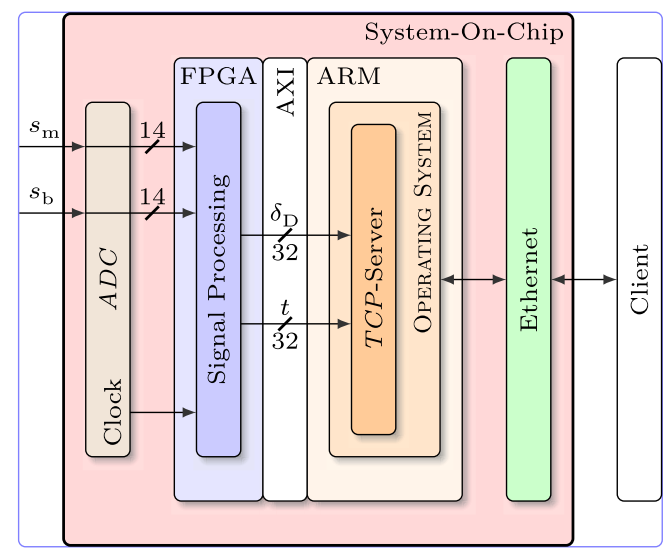

Fig. 10 Scheme of the signal processing and communication. The signals $s_{\mathrm{m}}$ and $s_{\mathrm{b}}$ from both SWSs are digitized and their phase difference $\delta_{\mathrm{D}}$ is calculated by the field programmable gate array (FPGA). $\delta_{\mathrm{D}}$ and a timestamp $t$ are transferred via an advanced extensible interface (AXI) to the operating system and from there distributed to the client via TCP

\section{Measurements}

Prior to the utilization in an optical standing wave, the sensors have intensively been investigated regarding their optical, electrical and electro-optical properties [19, 24, 26].

\subsection{Single Standing-Wave Sensor}

The HSWI was in a first place realized according to Fig. 4 to prove the basic function of the proposed interferometer type. The laser source for providing the two laser frequencies $f_{1}$ and $f_{2}$ consists of two PLL coupled He-Ne lasers according to Fig. 5, which are fiber coupled and connected to the actual interferometer. In the interferometer, two fiber collimators form the diametrically opposed laser sources, emitting towards each other. In between these collimators, the SWS is placed and the investigations in this section are made.

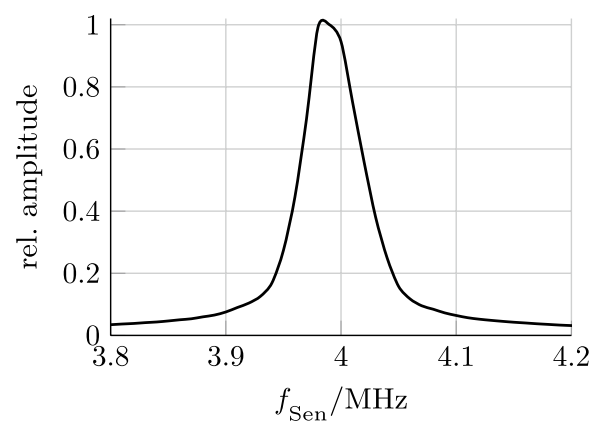

Fig. 11 Spectrum of the photo signal of a fixed SWS in an optical setup according to Fig. 4 at a beat frequency $f_{\mathrm{b}}=3.98 \mathrm{MHz}$ 


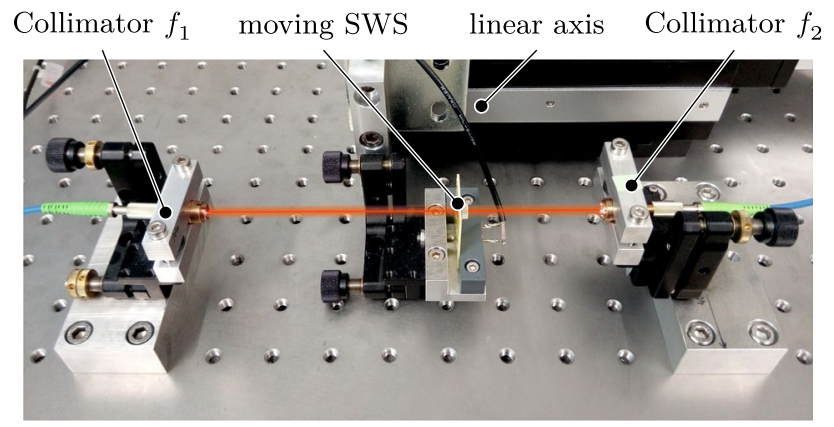

Fig. 12 Setup according to Fig. 4 with the SWS mounted to a motorized linear axis with a moving range of $100 \mathrm{~mm}$. The laser sources for $f_{1}$ and $f_{2}$ are fiber collimators, fed by the PLL coupled lasers described in Sect. 3.1

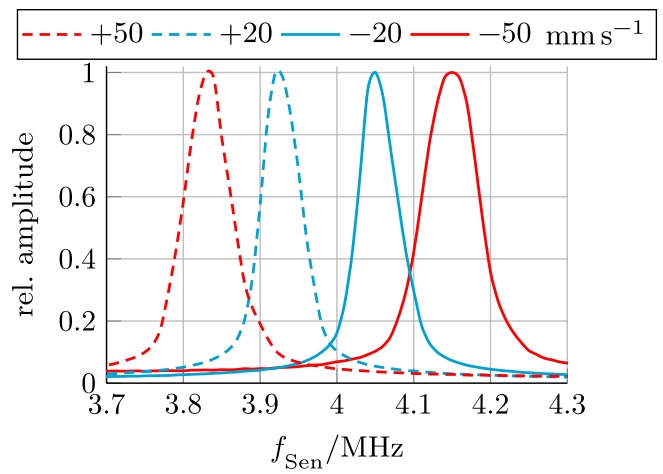

Fig. 13 Spectra of the photo signal of an SWS moving along the optical axis at different speeds $\dot{z}_{\text {Sws }}$ ranging from $-50 \mathrm{~mm} \mathrm{~s}^{-1}$ to $+50 \mathrm{~mm} \mathrm{~s}^{-1}$ in an optical setup according to Fig. 4. The motion of the SWS induces a direction and speed dependent Doppler shift of $f_{\text {Sen }}$

At first, the signal $s_{\mathrm{m}}$ of the SWS was recorded. From Eq. (10) it can be deduced that in this situation the sensor frequency $f_{\text {Sen }}$ should equal the beat frequency $f_{\mathrm{b}}$. Figure 11 shows the spectrum of the recorded signal.

As can be seen in Fig. 11, the frequency of a fixed SWS is $f_{\text {Sen }}=f_{\mathrm{b}}$. Furthermore, the exact beat frequency of the laser source (Fig. 5) is $3.98 \mathrm{MHz}$.

To verify the Doppler shift claimed in Eq. (9), the SWS was mounted on a motorized linear axis and moved with different velocities in $+z$ and $-z$ direction, thus with the motion of the standing wave ( $\dot{z}_{\mathrm{sW}}$, Figs. 2 and 3 ) and against it. The setup is shown in Fig. 12.

During the time period of constant velocity, the sensor signal was recorded. Figure 13 shows the resulting spectra for different velocities of the SWS.

As anticipated, the sensor frequency $f_{\text {Sen }}$ is shifted from the beat frequency $f_{\mathrm{b}}$ when moving the SWS along the optical axis. The sign and value of the measured $f_{\mathrm{D}}$ corresponds to Eq. (9), as can be seen when comparing Fig. 13 to Table 1.
Table 1 Doppler shift for a moving SWS in a setup according to Fig. 4 respectively 12, calculated using Eq. (9) and a beat frequency of $f_{\mathrm{b}}=3.98 \mathrm{MHz}$

\begin{tabular}{lll}
\hline$\dot{z}_{\text {SWS }} / \mathrm{mm} \mathrm{s}^{-1}$ & $f_{\mathrm{D}} / \mathrm{kHz}$ & $f_{\text {Sen }} / \mathrm{MHz}$ \\
\hline 50 & 158 & 3.82 \\
20 & 63.2 & 3.92 \\
-20 & -63.2 & 4.04 \\
-50 & -158 & 4.14 \\
\hline
\end{tabular}

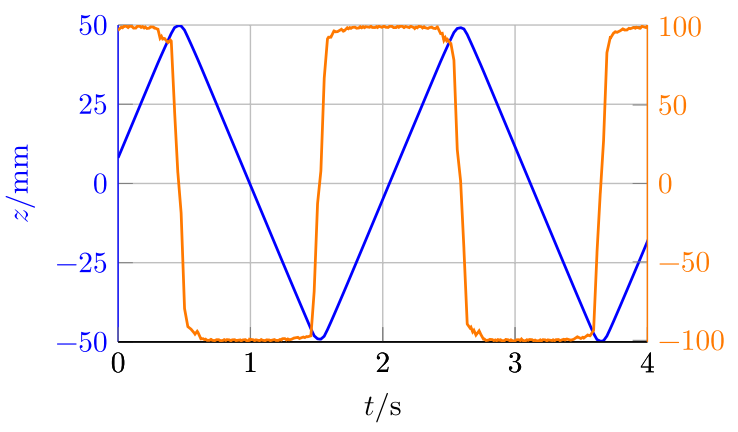

Fig. 14 Proof of the ability of the heterodyne interferometer for tracking mirror motions with high velocities over a large range. [24]

\subsection{Heterodyne Standing-Wave Interferometer}

After the basic investigations in the last section, a second SWS as depicted in Fig. 8 was inserted in the optical path. The second SWS was held at rest as a reference for the beat signal while several motions were carried out with the first sensor.

\subsubsection{Measuring velocity}

To prove the ability of the HSWI for high measuring velocities, the motorized linear axis from Sect. 5.1 was utilized again. This time, the SWS was driven with maximum velocity of $0.1 \mathrm{~m} \mathrm{~s}^{-1}$ over the whole positioning range of $100 \mathrm{~mm}$ of the axis. Meanwhile, the signals of both SWS were fed into the system-on-chip for signal processing (Figs. 9 and 10). The demodulated length and velocity information can be seen in Fig. 14.

\subsubsection{Resolution}

A major reason for using interferometric length measurement systems is their inherent high resolution in the nanometer range. To show the resolution of the developed HSWI, the measuring SWS was mounted to a piezo actuator and moved in nanometer steps back and forth along 


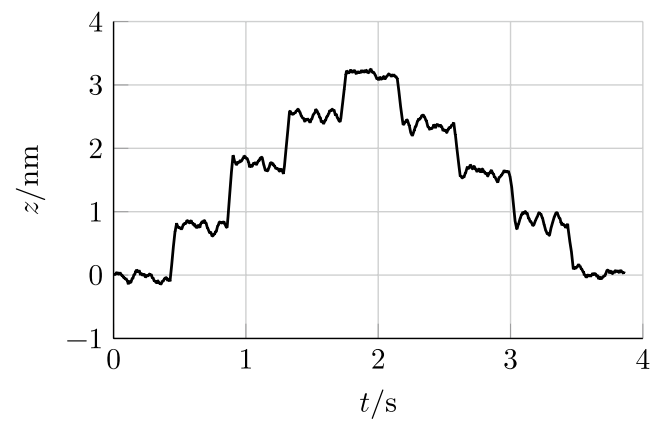

Fig. 15 Stepwise motion of the measuring SWS along the optical axis (sampling rate $233 \mathrm{~Hz}$ ). The mean step height is $0.8 \mathrm{~nm}$

the optical axis. Figure 15 shows the calculated position of the sensor.

\subsubsection{Periodic non-linearities}

Although interferometric measurement techniques offer resolutions down to the picometre range [25], the actual accuracy is, among other things, limited by periodic non-linearities of the length signal. For state-of-the-art Michelson-type heterodyne interferometers, these non-linearities originate from frequency and polarization mixing, misalignment of optical parts, etc. For the HSWI, these considerations are not applicable, as there are no additional optical parts except the SWSs, no reference and measuring arm in the classical sense, and hence no undesired frequency mixing. However, due to the working principle of a standing-wave interferometer, the SWSs need to be aligned exactly perpendicularly to the optical axis [19]. For that reason, the SWSs in an HSWI are almost perfectly parallel to each other and form an optical resonator, resulting in multi-beam interferences, harmonics, and hence periodic non-linearities.

To quantify the non-linearities of a measurement system, an ideal motion, itself free from any periodic non-linearities, is required. Measuring such an ideal motion with the interferometer should result in an ideal, linear length signal. Any remaining non-linearities can then be assigned to the laser source, the interferometer, and the signal processing. Such motion was realized by a simple heated brass rod, using its thermal expansion as the actuation for the measuring SWS. In theory, the thermal expansion follows an exponential increase or decline and is assured to be free from periodic non-linearities, especially at integer fractions of the laser wavelength $\lambda$.

In the experiment, the measuring SWS was mounted to the brass rod, which was heated to a certain temperature. After the heating was switched off, the length signal of the HSWI was recorded, eliminating any influence from the electrical supply for the heating. Afterwards, the length of the signal was approximated by an exponential function and

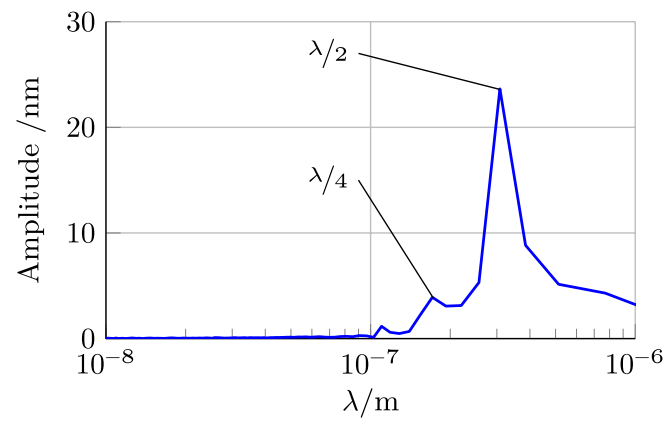

Fig. 16 Periodic non-linearities of the developed HSWI. The major peak at $\lambda / 2=316 \mathrm{~nm}$ is caused by multi-beam interferences between the parallel SWSs and the cross talk between the analogue channels of the signal processing unit [24]

the residuals were calculated. Figure 16 shows the corresponding spectrum.

As can be seen in Fig. 16, the major periodic non-linearities are at the half laser wavelength with an amplitude of $22 \mathrm{~nm}$. As stated above, these are mainly caused by an imperfect anti-reflection coating of the SWSs [24].

\section{Conclusions}

In this paper, a heterodyne standing-wave interferometer with improved phase stability is proposed. Heterodyne standing waves arise between two diametrically opposed laser sources of different wavelength. The counter propagating beams interfere in opposite directions and form a periodic intensity profile moving along the optical axis with a constant velocity. The sensors for detecting this intensity profile are transparent and have a thickness of only $40 \mathrm{~nm}$, so they do not interfere with the standing wave. When a single sensor is used inside the standing-wave interferometer, phase deviations of the laser source directly lead to length measurement errors. Therefore, in this paper a second sensor is included in the optical setup, serving as a reference for the current phase angle of the standing wave and this way improving the phase stability of the interferometer. The reference sensor is permanently fixed, providing a stable signal, which the signal of the moving sensor can be compared to. Furthermore, an algorithm is developed to allow for a flexible signal demodulation and processing of the reference and the measuring signal. Finally, different experiments are made to show the parameters of the developed interferometer type like dynamic properties, resolution, and periodic non-linearities.

While the resolution of the system as well as the dynamic behavior is excellent and should be sufficient for many length measurement applications, the non-linearities will be subject to further investigations in the future. The main 
parameter to improve is the reflectivity of the sensors, which should ideally be zero but currently is in the range of $10 \%$.

Author Contributions Conceptualization: E. M., I. W. R.; Supervision: E. M., I. W. R., J.-P. Z.; Sensor fabrication: J.-P. Z.; Investigation and Realization of the Measurements: I. O.; Software coding: I. O.; Analysis of the data: I. O.; Writing - original draft preparation: I. O.; Writing - review and editing: J. S., E. M.

Funding Open Access funding enabled and organized by Projekt DEAL.. The project was funded by the German Federal Ministry of Education and Research under contract 03V0235.

Availability of data and material All original data are available upon request from the corresponding author.

Code availability Software code is available upon request from the corresponding author, depending on the original software licence / EULA.

\section{Declarations}

Conflict of interest The authors declare that they have no conflicts of interest.

Open Access This article is licensed under a Creative Commons Attribution 4.0 International License, which permits use, sharing, adaptation, distribution and reproduction in any medium or format, as long as you give appropriate credit to the original author(s) and the source, provide a link to the Creative Commons licence, and indicate if changes were made. The images or other third party material in this article are included in the article's Creative Commons licence, unless indicated otherwise in a credit line to the material. If material is not included in the article's Creative Commons licence and your intended use is not permitted by statutory regulation or exceeds the permitted use, you will need to obtain permission directly from the copyright holder. To view a copy of this licence, visit http://creativecommons.org/licenses/by/4.0/.

\section{References}

1. Büchner H (1983) Stehende-Wellen-Interferometer zur Messung von optischen Gangunterschieden. DE 3300369:C2

2. Bunte E, Mandryka V, Jun K, Büchner H-J, Jäger G, Stiebig H (2004) Thin transparent pinphotodiodes for length measurements. In: Sensors and actuators a: physical, vol 113, p 3

3. Hecht E (2009) Optik. 5th ed. Oldenbourg Wissenschaftsverlag $\mathrm{GmbH}$

4. Büchner H, Stiebig H, Mandryka V, Bunte E, Jäger G (2003) An optical standing-wave interferometer for displacement measurements. In: Measurement science and technology, vol 14, p 3. https://doi.org/10.1088/0957-0233/14/3/309

5. Ortlepp I, Manske E, Zöllner J-P, Rangelow I (2018) Heterodyne standing-wave interferometer. In: tm - Technisches Messen. Plattform für Methoden, Systeme und Anwendungen der Messtechnik, vol 85, p 1. https://doi.org/10.1515/teme-2018-0017

6. Zeeman P (1896) Ueber einen Einfluss der Magnetisirung auf die Natur des von einer Substanz emittirten Lichtes. In: Verhandlungen der Physikalischen Gesellschaft zu Berlin

7. Bragg WH, Bragg WL (1913) The reflection of X-rays by crystals. In: Proceedings of the Royal Society of London. Series A, Containing Papers of a Mathematical and Physical Character, vol 88, p 605. https://doi.org/10.1098/rspa.1913.0040
8. White A, Rigden J (1962) Continuous gas maser operation in visible. Proc Inst Radio Eng 50(7):1697

9. Yu-Ye J, Shu-Lian Z, Yan L, Ji-Hua G, Jia-Qiang L (2001) Zeeman-Birefringence He-Ne Dual Frequency Lasers. In: Chinese Physics Letters, vol 18, p 4. https://doi.org/10.1088/0256-307x/ $18 / 4 / 322$

10. Weichert C, Köchert P, Köning R, Flügge J, Andreas B, Kuetgens U, Yacoot A (2012) A heterodyne interferometer with periodic nonlinearities smaller than $\pm 10 \mathrm{pm}$. In: Measurement Science and Technology, vol 23, p 9. https://doi.org/10.1088/0957-0233/23/9/ 094005

11. Kim M-S, Kim S-W (2002) Two-longitudinal mode He-Ne laser for heterodyne interferometers to measure displacement. In: Appl. Opt. vol 41, p 28. https://doi.org/10.1364/AO.41.005938

12. Ellis JD, Meskers AJ, Spronck JW, Schmidt RHM (2011) Fibercoupled displacement interferometry without periodic nonlinearity. Optics Lett 36(18):3584-3586. https://doi.org/10.1364/ol.36. 003584

13. Knarren B, Cosijns S, Haitjema H, Schellekens P (2005) Validation of a single fibre-fed heterodyne laser interferometer with nanometre uncertainty. Precis Eng 29(2):229-236. https://doi.org/ 10.1016/j.precisioneng.2004.09.003

14. Sternkopf C, Diethold C, Gerhardt U, Wurmus J, Manske E (2012) Heterodyne interferometer laser source with a pair of two-phase locked-loop coupled He-Ne lasers by $632.8 \mathrm{~nm}$. In: Measurement Science and Technology, vol 23, p 7. https://doi.org/10.1088/ 0957-0233/23/7/074006

15. Sternkopf C, Wurmus J, Gerhardt U, Manske E (2012) Fiber coupled plane mirror heterodyne laser interferometer with two-phase locked-loop coupled He-Ne lasers. In: 16th international conference on mechatronics technology, pp 16-19

16. Tilsch J (1994) Device for frequency-stabilisation of He-Ne internal mirror lasers. DE 4303217 A1, p 11

17. Sternkopf C, Göllner S, Manske E (2014) Frequency stabilization of an external-cavity diode laser by offset frequency looking to a stabilized $\mathrm{He}-\mathrm{Ne}$ laser. In: SPIE Photonics Europe. International Society for Optics and Photonics. https://doi.org/10.1117/ 12.2051521

18. Wiener O (1890) Stehende Lichtwellen und die Schwingungsrichtung polarisirten Lichtes. In: Annalen der Physik, vol 276, p 6. https://doi.org/10.1002/andp.18902760603

19. Ortlepp I, Büchner H-J, Ivanov T, Hofer M, Zöllner J-P, Rangelow I, Manske E (2015) Ultrathin transparent photodetector for use in standing-wave interferometer. In: XXI Imeko world congress full papers (Prague, Aug. 30, 2015-Sept. 4, 2015). Ed. by Holub, $\mathrm{J}$

20. Silvertooth EW, Jacobs SF (1983) Standing wave sensor. In: Applied optics, vol 22, p 9

21. Sasaki M, Mi X, Hane K (1999) Standing wave detection and interferometer application using a photodiode thinner than optical wavelength. In: Applied Physics Letters, vol 75, p 14. https://doi. org/10.1063/1.124898

22. Mandryka V (2009) Entwicklung, Aufbau und Untersuchung eines Stehende-Wellen-Interferometers. PhD thesis. Technische Universität Ilmenau

23. Ortlepp I, Manske E, Zöllner J-P, Rangelow IW (2020) Phasemodulated standing wave interferometer. In: (Graz, Nov. 4, 2020). p 30

24. Ortlepp I (2020) Mikrointerferometer auf Basis von interferenzoptischen Stehende - Welle - Sensoren. Dissertation. Technische Universität Ilmenau

25. Köchert P, Flügge J, Weichert C, Köning R, Manske E (2012) Phase measurement of various commercial heterodyne $\mathrm{He}-\mathrm{Ne}-$ laser interferometers with stability in the picometer regime. In: Measurement Science and Technology, vol 23, p 7 
26. Ortlepp I, Büchner H-J, Ivanov T, Hofer M, Zöllner J-P, Rangelow I, Manske E (2016) Miniatur-Stehende-Welle-Interferometer auf Basis schneller, transparenter Photodioden. In: AMA Service
GmbH, Von-Münchhausen-Str. 49, 31515 Wunstorf, Germany. https://doi.org/10.5162/sensoren2016/6.1.2 